\title{
Expression and regulation of caudal in the lower cyclorrhaphan fly Megaselia
}

\author{
Michael Stauber • Steffen Lemke • Urs Schmidt-Ott
}

Received: 13 November 2007 / Accepted: 9 January 2008 /Published online: 24 January 2008

(C) Springer-Verlag 2008

\begin{abstract}
The homeobox gene caudal ( $c a d$ ) regulates posterior development in Drosophila. In early embryos, the cad protein (CAD) is expressed in a posterior-to-anterior concentration gradient, which contributes polarity to the developing embryo. The CAD gradient is complementary to and dependent on the anterior pattern organizer Bicoid (BCD), which represses the translation of ubiquitous maternal cad transcripts in the anterior embryo through a direct interaction with the cad 3' untranslated region (UTR). Here, we show that early embryos of the lower cyclorrhaphan fly Megaselia express the putative $c a d$ orthologue Mab-cad throughout the posterior three quarters of the blastoderm but lack maternal transcripts. In transgenic blastoderm embryos of Drosophila, Mab-cad cis-regulatory DNA drives the expression of a reporter gene in a similar pattern, while Mab-cad 3' UTR fails to mediate translational repression of a ubiquitously transcribed reporter. For another lower cyclorrhaphan fly (Lonchoptera) and two related outgroup taxa of Cyclorrhapha
\end{abstract}

Communicated by P. Simpson

M. Stauber $\cdot$ U. Schmidt-Ott

Max-Planck-Institut für Biophysikalische Chemie,

Abt. Molekulare Entwicklungsbiologie,

Am Fassberg 11,

37077 Göttingen, Germany

S. Lemke $\cdot$ U. Schmidt-Ott $(\bowtie)$

University of Chicago,

Dept. of Organismal Biology and Anatomy,

CLSC 921B, 920 E. 58th Street,

Chicago, IL 60637, USA

e-mail: uschmid@uchicago.edu

Present address:

M. Stauber

Cancer Research UK, Developmental Genetics Laboratory,

44 Lincoln's Inn Fields,

London WC2A 3PX, UK
(Empis, Haematopota), we report maternal cad expression in ovarian follicles. Together, our results suggest that BCD is not required for the translational repression of Mab-cad, and that maternal cad expression was lost in the Megaselia lineage.

Keywords Gene expression · Enhancer .

Translational repression $\cdot$ Evolution $\cdot$ Brachycera

\section{Introduction}

The protein products of the homeobox gene cad (CAD) accumulate in a concentration gradient across early insect embryos and are an important factor of posterior patterning. cad-deficient Drosophila embryos exhibit severe defects in the abdomen and thorax (Macdonald and Struhl 1986; Wu and Lengyel 1998), and cad RNA interference (RNAi) in short-germ insects (Tribolium, Gryllus) yielded embryos that consist of the prognathal head only (Copf et al. 2004; Shinmyo et al. 2005). In Drosophila, the full complement of embryonic cad activity requires maternal and zygotic transcripts (Macdonald and Struhl 1986). Homozygous $\mathrm{cad}^{-}$ embryos from heterozygous mothers only lack terminal structures including anal tufts, parts of the anal pads, and posterior sensory organs. In these embryos, maternal cad expression is sufficient for the proper formation of segments. Conversely, ectopic cad expression in the anterior embryo causes head defects (Mlodzik et al. 1990; Niessing et al. 1999; Niessing et al. 2000; Cho et al. 2005).

The CAD gradient of Drosophila forms by translational repression of ubiquitous early cad transcripts in the anterior embryo, and is mediated by the protein products of bicoid (BCD) and d4EHP (Dubnau and Struhl 1996; RiveraPomar et al. 1996; Cho et al. 2005). The current model of 
this mechanism proposes that ubiquitous d4EHP binds to the $5^{\prime}$ cap structure of $c a d$ mRNA and to BCD, which also directly binds to a $\mathrm{BCD}$ binding region (BBR) in the $3^{\prime}$ UTR of $c a d$ mRNA, thereby circularizing the transcript and preventing translation initiation (Niessing et al. 2002; Cho et al. 2005). In other insects, a mechanism of CAD gradient formation has not been elucidated. In the parasitic wasp Nasonia, cad transcripts are localized at the posterior pole and a CAD protein gradient may be formed by diffusion (Olesnicky et al. 2006). In the silk moth, Bombyx, and in the red flour beetle, Tribolium, translational repression of ubiquitous maternal cad transcripts occurs in the anterior embryo but these insects, like Nasonia, lack a BCD orthologue (Xu et al. 1994; Schulz et al. 1998).

Here, we report data on the expression and regulation of a cad homologue (Mab-cad) from the lower cyclorrhaphan fly Megaselia abdita (Phoridae). We show that Mab-cad expression in the blastoderm is similar to the expression of cad in the higher cyclorrhaphan fly Drosophila. Unlike cad, however, Mab-cad is not expressed in ovarian follicles, and early embryos therefore lack maternal transcripts. A lacZ reporter construct containing ca. $8 \mathrm{~kb}$ of Mab-cad cisregulatory DNA upstream of the first Mab-cad exon essentially recapitulates the endogenous early Mab-cad pattern in transgenic Drosophila, while the Mab-cad 3' UTR fails to suppress the anterior translation of a ubiquitously transcribed reporter gene. Finally, we report the expression of cad homologues in the ovarian follicles of three related dipterans (Lonchoptera, Empis, and Haematopota). Our results suggest the loss of maternal cad expression in the Megaselia lineage and that $\mathrm{BCD}$ is not required for the translational repression of Mab-cad.

\section{Materials and methods}

Sample collection

M. abdita Schmitz (Phoridae) was obtained from our laboratory culture. Adults of Empis livida L. (Empididae), Haematopota pluvialis L. (Tabanidae), and Lonchoptera lutea Panzer (Lonchopteridae) were caught in the surroundings of Göttingen (Germany).

\section{Cloning procedures}

Partial homeobox sequences of cad homologues were isolated by polymerase chain reaction (PCR) using the

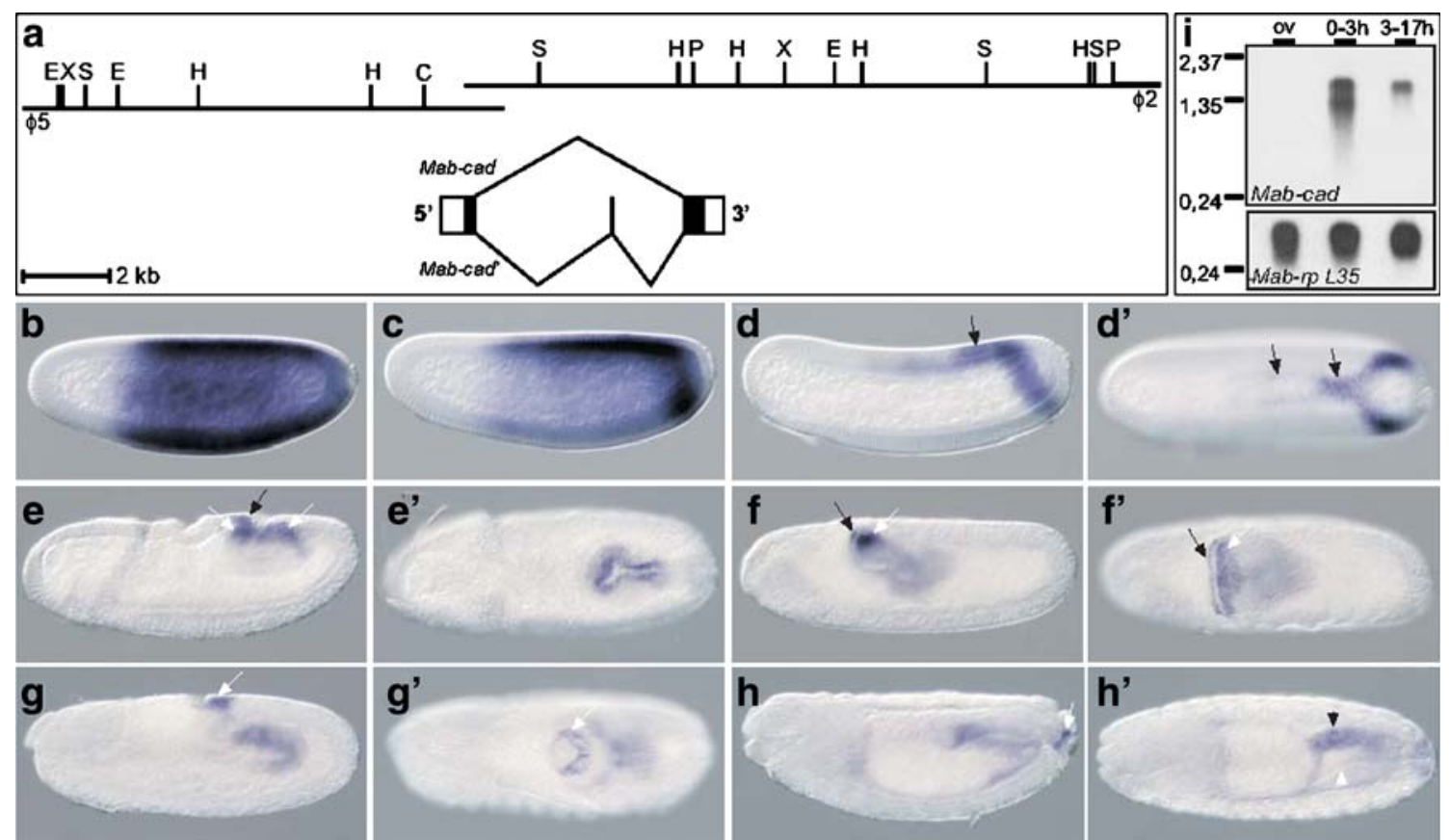

Fig. 1 Characterization of Mab-cad. a Genomic organization of the Mab-cad locus. Restriction sites of ClaI $(C), E c o$ RI $(E), H i n d I I I ~(H)$, PstI $(P)$, SpeI $(S)$, and $X b a \mathrm{I}(X)$ on two phage clones $(\Phi 5, \Phi 2)$ are shown above alternative splice variants of $M a b-c a d$. ORF sequence is shown as filled boxes. Mab-cad expression is shown at syncytial blastoderm (b), blastoderm cellularization (c), cellular blastoderm (d, $\mathbf{d}^{\prime}$ ), germband extension $\left(\mathbf{e}-\mathbf{f}^{\prime}\right)$, germband retraction $\left(\mathbf{g}, \mathbf{g}^{\prime}\right)$ stage, and shortly before dorsal closure $\left(\mathbf{h}, \mathbf{h}^{\prime}\right)$. All embryos are shown in lateral view with anterior to the left. Embryos at later stages are also shown in dorsal view (d', $\left.\mathbf{e}^{\prime}, \mathbf{f}^{\prime}, \mathbf{g}^{\prime}, \mathbf{h}^{\prime}\right)$. Amniotic expression (black arrows), posterior embryonic expression (white arrows), and posterior midgut expression (arrowhead) relative to the position of the unstained hindgut (white arrowheads) are indicated. i Northern Blot with poly (A) RNA from ovaries (ov), early $(0-3 h)$ and late (3-17 $h$ ) embryos hybridized with Mab-cad probe (top) and subsequently with a MabrpL35 probe (bottom). RNA standard sizes in $\mathrm{kb}$ are shown at the left margin 
degenerate primer pair 5'AAR ACS CGB ACS AAR GAY AA / 5'VCG VCG RTT YTG RAA CCA RAT on genomic DNA. 5' and $3^{\prime}$ rapid amplification of cDNA ends (RACE) reactions were performed using MARATHON and SMART cDNA Amplification Kits (Clontech) starting with ovarial (Empis, Haematopota), embryonic (Megaselia), and adult (Lonchoptera, Megaselia) poly $\left(\mathrm{A}^{+}\right)$RNA. A Megaselia riboprotein $\mathrm{L} 35$ gene fragment was accidentally recovered in a PCR with degenerate primers and used as a positive control in the Northern Blot analysis. All sequences have been deposited under EU295452-EU295458 in the GenBank database.

Two genomic phages, which include the Mab-cad locus, were obtained from a Lambda-Fix II (Stratagene) phage library of adult flies (unpublished). Genomic DNA for the reporter construct was PCR amplified using TaKaRa LA Taq polymerase (TaKaRa) on Lambda-Fix II phage clone 5 using 5' TTT CGA TTG CAC TAA ATA A and M13 $(-20)$. The product was cloned into pGEM-T Easy (Promega), excised with NotI and cloned into pCaSpeRhs43-lacZ (Thummel and Pirrotta 1992). A clone with the insert in normal orientation relative to the transcription start was used for transgenic experiments.

To assay translational control in the Drosophila blastoderm, we generated a construct containing the Mab-cad open reading frame (ORF; $981 \mathrm{bp}$ including start and stop codon) with an N-terminal hemagglutinin (HA)-tag (ATG TAC CCA TAC GAT GTG CCA GAT TAC GCC) as reporter gene. At the $5^{\prime}$ end, the reporter gene was flanked by the maternal hunchback promoter (nucleotides 1 to 1512 of GenBank accession Y00274; Tautz et al. 1987). At the 3' end, AscI, MluI and FseI cloning sites and the SV40 small t antigen 3' UTR (853 bp) were added. The cassette was cloned into the vector pCaSpeR4 (Thummel and Pirrotta 1992) using BamH1. Drosophila cad 3' UTR (856 bp), truncated cad 3' UTR downstream of the BBR (343 bp) and Mab-cad 3' UTR (559 bp) were PCR amplified with the following primer pairs, respectively: 5'-GGC GCG CCA CGA CCA TTC CTG TTA TGC / 5'-GGC CGG CCG AGT TGC TTT ATC TAT GGT G, 5'-GGC GCG CCG TTA AAC ATA ATT TTA GTA CAA GC / 5'-GGC CGG CCG AGT TGC TTT ATC TAT GGT G, and 5'-GGC GCG CCA TAT TGG GAG AAC CAC GTC / 5'-GGC CGG CCG AAT GAT TAA AAA TTT TAT TTT TCA TCG. These primers contain AscI (upper primers) or FseI (lower primers) endonuclease restriction sites that were used for directional cloning of the $3^{\prime}$ UTRs into the reporter cassette.

In situ and Northern hybridization and antibody staining

In situ and Northern hybridizations were done as described (Stauber et al. 2002; Rafiqi et al. 2008). Digoxigeninlabeled RNA probes were synthesized from 5' (Empis, Lonchoptera), or 3' cad RACE products (Megaselia,
Haematopota) and from the complete ORF (lacZ). Transcripts were detected using anti-digoxigenin-AP antibody (Roche) and NBT/BCIP (Roche). HA was detected using anti-HA rat monoclonal antibody (Roche) and standard Drosophila protocols.

\section{Results and discussion}

Genomic organization and expression of a cad homologue from Megaselia

A homeobox fragment of Megaselia caudal (Mab-cad) was obtained by degenerate PCR. This sequence was extended by $5^{\prime}$ and $3^{\prime}$ RACE experiments on cDNA templates from both embryos and adult flies. We obtained two transcripts, which differed in the presence of a small (54 bp) exon in
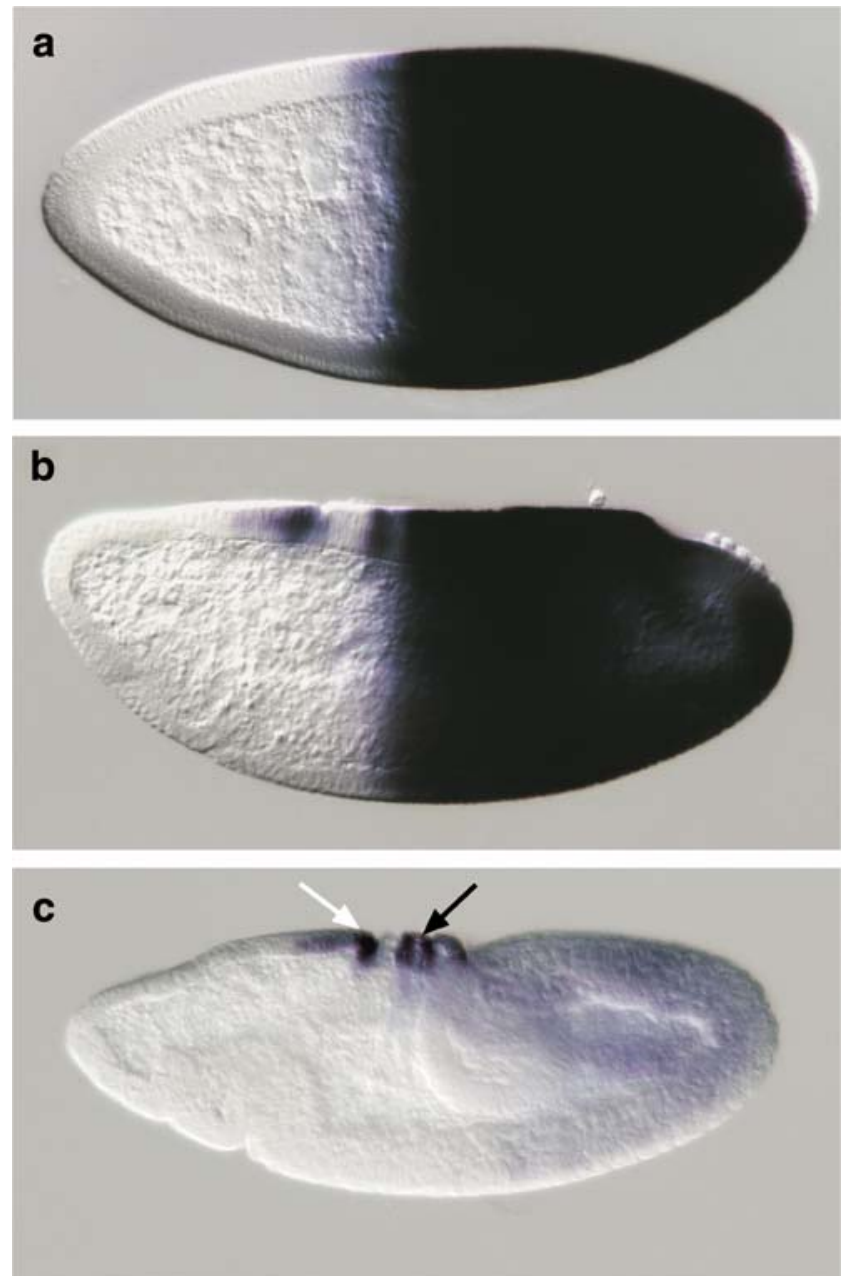

Fig. 2 Expression of lacZ transcripts under the control of Mab-cad cis-regulatory DNA in transgenic Drosophila. Embryos are shown at a blastoderm, b early gastrulation, and $\mathbf{c}$ germband extension. Anterior is left and dorsal up. The white arrow designates expression in the dorsal portion of the cephalic furrow and the black arrow expression in the posterior amnioserosa 
the open reading frame of the embryonic transcript. Both Megaselia transcripts were mapped onto phage clones from a representative genomic library of Megaselia, revealing two alternative splice variants of the same locus (Fig. 1a; we refer to the sequence without the $54 \mathrm{bp}$ exon as Mab$c a d$ and to the sequence with this exon as Mab-cad'). The position of the miniexon is identical to the position of a miniexon that has been reported as an alternative splice variant of the Tribolium cad transcripts $T c-c a d A$ and $T c$ cadB (Schulz et al. 1998). In Drosophila, this splice variant may have been lost. The predicted full-length proteins of $M a b-c a d$ and Mab-cad' share $38 \%$ and $34 \%$ sequence similarity, respectively, with the predicted CAD protein of Drosophila. The homeodomains of Mab-CAD and CAD are $92 \%$ identical. These data suggest that the Mab-cad locus is orthologous to the cad locus of Drosophila.

Embryonic whole mount in situ hybridization experiments with Mab-cad probes against the 5' exon (not shown) and the $3^{\prime}$ exon (Fig. 1b-h') gave identical patterns. Zygotic expression starts one nuclear cycle prior to the onset of cellularization and occurs throughout the posterior $75 \%$ of the blastoderm. During blastoderm cellularization, Mab-cad expression changes rapidly and becomes restricted to the amnion anlage (black arrows in Fig. 1; Rafiqi et al. 2008) and a narrow posterior stripe (Fig. 1c-d'). During germband extension, the posterior expression marks the end of the germband, and the amniotic expression becomes restricted to a narrow stripe that invaginates with the proctodeum (Fig. 1e-g'). In addition, a new expression domain appears in the posterior midgut. This expression is strongest in the narrow posterior portion of the midgut, which bends dorsoanterior and connects to the hindgut, which does not express Mab-cad. In later embryos, we detected Mab-cad expression in this domain and in a ring around the posterior hindgut (Fig. 1h,h'). Unlike cad in Drosophila (Macdonald and Struhl 1986), Mab-cad is not expressed in the pole cells, malpighian tubules, and bilateral pairs of neurons. Also, we could not detect Mab-cad transcripts in ovaries and very early embryos, indicating that embryos lack maternal transcripts. To confirm this result, we examined the expression of $M a b-c a d$ in a developmental Northern blot. This experiment revealed two transcripts in $0-3 \mathrm{~h}$ old embryos (Fig. 1i). The longer transcript was also detected at later stages and matches the predicted length of Mab-cad (with and without the mini exon). In ovaries, the detection of residual amounts of the longer Mab-cad transcript required a 16 times longer exposure time than the detection of much stronger bands in embryos (not shown), confirming the

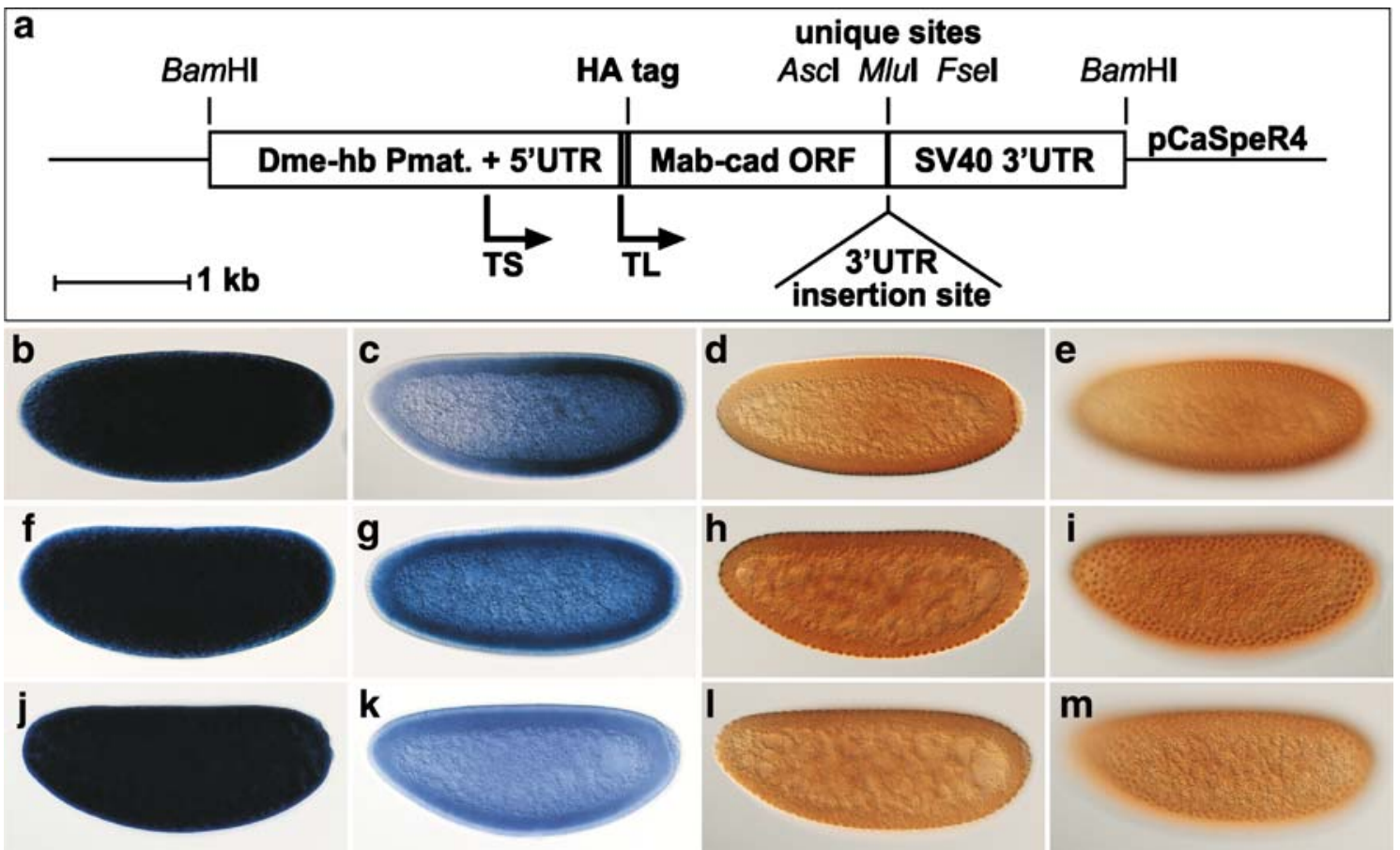

Fig. 3 Translational repression assay. a Schematic overview of the translation assay cassette. $T S$, transcription start, $T L$ translation start. Transgenic Drosophila embryos containing the translation assay cassette with the complete Drosophila cad 3' UTR (b-e), a truncated Drosophila cad 3' UTR lacking the BBR (f-i), or the Mab-cad 3' UTR (j-m) are shown. Mab-cad in situ hybridizations of pre-blastoderm $(\mathbf{b}, \mathbf{f}, \mathbf{j})$ and blastoderm embryos (c, $\mathbf{g}, \mathbf{k})$ as well as two focal planes of early blastoderm embryos stained with a hemagglutinin $(H A)$ antibody (d, e, $\mathbf{h}, \mathbf{i}, \mathbf{l}, \mathbf{m})$ are shown for each of the three transgenic lines. Note that in

blastoderm embryos with the complete Drosophila cad 3' UTR Mabcad transcripts disappear from the anterior pole (c) coincident with translational repression of the reporter $(\mathbf{d}, \mathbf{e})$. The distribution of $\mathrm{Mab}$ cad transcripts and protein remains uniform in embryos carrying the cassette with the truncated Drosophila cad $3^{\prime}$ UTR $(\mathbf{g}-\mathbf{i})$, or the Mabcad 3' UTR $(\mathbf{k}-\mathbf{m})$. In situ hybridizations with an SV40 anti-sense probe were negative, indicating that the polyadenylation signals (AAUAAA) of the added $c a d$ and Mab-cad 3' UTR sequences are functional. All embryos are shown with anterior to the left and dorsal up 
absence of significant maternal Mab-cad transcription in nurse cells and oocytes.

\section{Regulation and evolution of Mab-cad expression}

The lack of maternal Mab-cad expression suggests that in Megaselia, transcriptional regulation might be sufficient for the anterior repression of Mab-cad in the early blastoderm. To test this prediction, we cloned $8 \mathrm{~kb}$ immediately upstream of the identified Mab-cad transcript sequence into a $l a c Z$ reporter vector and examined the expression of $l a c Z$ under the control of Mab-cad cis-regulatory DNA in transgenic blastoderm embryos of Drosophila. Two independent transgenic lines expressed lacZ in patterns reminiscent of endogenous Mab-cad expression (Fig. 2a; a third transgenic line showed no expression). Unlike early zygotic transcripts of endogenous cad, which accumulate in two broad stripes (Schulz and Tautz 1995), the transcripts of the transgene accumulated evenly throughout the posterior blastoderm like Mab-cad transcripts in Megaselia. Compared to Mab-cad expression in Megaselia, the anterior expression boundary of the transgene was shifted by about $10 \%$ egg length towards posterior. In addition, the transgene was expressed in the dorsal portion of the cephalic furrow and in the posterior portion of the amnioserosa (Fig. 2b,c). These observations are consistent with the hypothesis that the anterior repression of Mab-cad activity

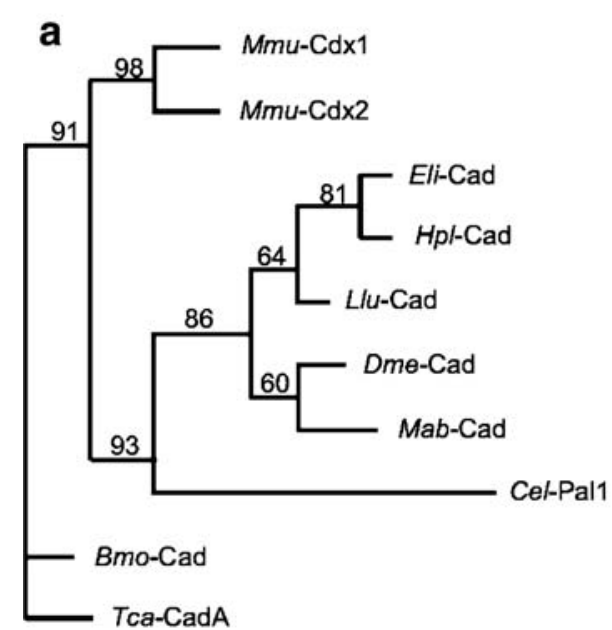

0.1 changes

Fig. 4 Homeodomain tree of CAD homologues and expression in ovarian follicles. (a) Quartet Puzzling analysis (Strimmer and von Haesseler 1996) with sequences from the dipterans E. livida (Eli), $H$. pluvialis (Hpl), L. lutea (Llu), M. abdita (Mab) and Drosophila melanogaster (Dme; accession no. NP 476954), the silk moth Bombyx mori (Bmo; NP_001037514), Tribolium castaneum (Tca; NP 001034498), the nematode Caenorhabditis elegans (Cel; NP 001021210$)$ and the mouse (Mmu; Cdx-1: AAA37412; Cdx-2: AAA19645). The tree has been constructed with the homeodomain sequences using the default settings of software on http://bioweb. pasteur.fr/. Support for internal branches is shown in percent. Branch

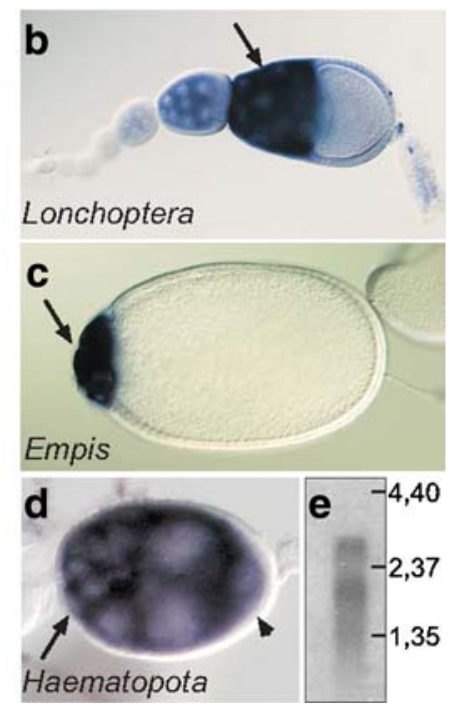

in early Megaselia embryos is regulated at the transcriptional level, without BCD-dependent posttranscriptional regulatory input.

Surprisingly, BCD represses the translation of a ubiquitously expressed cad transgene from Tribolium (Wolff et al. 1998). The signal provided by the transcript of this cad homologue that mediates translational repression in transgenic Drosophila has not been identified, but the observation raises the question whether $\mathrm{BCD}$ can repress the translation of a broad range of cad homologues. To test the effect of $\mathrm{BCD}$ on the translation of maternally expressed Mab-cad, we generated a reporter cassette to test 3' UTRs for their ability to mediate translational repression in early Drosophila embryos. The cassette contains the maternal promoter and 5' UTR of the segmentation gene hunchback, the hemagglutinin (HA)-tagged Mab-cad open reading frame, and a cloning site for 3' UTRs (Fig. 3a). To ensure the stability of reporter transcripts, SV40 3' UTR was included downstream of the multiple cloning site. Using this cassette, we generated constructs containing the cad 3' UTR from Drosophila, either with or without the Bicoid Binding Region (BBR; truncation 5' of BBR) or the cad 3' UTR from Megaselia, and tested their expression, both at the transcript and at the protein level in transgenic blastoderm embryos of Drosophila. In embryos containing the reporter construct with the complete 3' UTR of Drosophila cad, early reporter transcripts were evenly distributed. However, the translation of

lengths indicate the average number of amino acid changes per position (see scale). In situ hybridizations of ovarian follicles from $L$. lutea (b), Empis livida (c) and H. pluvialis (d), with anti-sense probes against the respective cad homologues. Expression is detected in the nurse cells (arrows) of Lonchoptera and Empis follicles, and in the nurse cells and the oocyte (arrowhead) of the Haematopota follicle. Note that the chorion may have prevented cad transcript detection in the oocytes of Lonchoptera and Empis. e Northern blot of poly(A) RNA from ovarian follicles of Haematopota hybridized with a radiolabeled Haematopota cad probe. RNA standard sizes are given in $\mathrm{kb}$ 
the reporter was repressed in the anterior quarter of the blastoderm, and in late blastoderm stages, the reporter transcript had disappeared from the anterior pole (4 independent lines; Fig. 3b-e). These observations match with the sequence of events in Drosophila, in which translational repression of ubiquitous cad transcripts at the anterior pole precedes the disappearance of anterior transcripts (own observations; cf. Fig. 5e-h, and 7a, c, e in Mlodzik and Gehring 1987), and suggests that translational repression mediated by the cad 3' UTR causes transcript instability. The reporter construct with the truncated 3' UTR of Drosophila cad expressed the reporter transcript and protein throughout the blastoderm (three independent lines; Fig. 3f-i). Likewise, the reporter construct with the 3' UTR of Mab-cad expressed the reporter transcript and protein throughout the blastoderm, albeit at a lower level (three independent lines; Fig. 3j-m). These results suggest that, unlike the $3^{\prime}$ UTR of cad, the $3^{\prime}$ UTR of Mab-cad is insufficient to mediate BCD-dependent translational repression in Drosophila.

Finally, we tested whether the absence of maternal cad expression is the ancestral condition for cyclorrhaphan flies, i.e. a plesiomorphic trait of Cyclorrhapha. We cloned cad homologues from a second lower cyclorrhaphan fly (L. lutea, Lonchopteridae), and two paraphyletic lower brachyceran taxa (H. pluvialis, Tabanidae; E. livida, Empididae) (Fig. 4a), and tested ovaries for maternal expression by in situ hybridization (Fig. 4b-d). In all three species, the nurse cells of ovarian follicles expressed the respective cad homologue. In Haematopota, we detected cad transcripts also in the oocyte (Fig. 4d). Failure to detect cad transcripts in the oocytes of Lonchoptera and Empis follicles is most likely a staining artefact due to the developing eggshell. For Haematopota, we verified maternal expression in a Northern blot and detected two cad transcripts (Fig. 4e), like in Megaselia (Fig. 1i) and Drosophila (Mlodzik and Gehring 1987), suggesting that the occurrence of two cad transcripts is conserved in evolution. However, the developmental time course of their appearance is not. In Drosophila, alternative cad promoters express the shorter transcript $(2.4 \mathrm{~kb})$ during oogenesis and the longer transcript $(2.6 \mathrm{~kb})$ during blastoderm cellularization (Mlodzik and Gehring 1987), while in Megaselia, the two cad mRNAs are transcribed during early embryogenesis. We cannot exclude that a paralogue of Mabcad provides maternal expression, but a very recent duplication of Mab-cad is unlikely because stringent hybridization of endonuclease digested genomic DNA with a Mab-cad probe in Southern blots is consistent with a single copy gene (not shown). Taken together, our results suggest that maternal cad expression was lost in the Megaselia lineage and that $\mathrm{BCD}$ is not required for the translational repression of Mab-cad.
Acknowledgements We thank Herbert Jäckle (Max-Planck-Institut für Biophysikalische Chemie, Göttingen) for support. Funding was provided by the Deutsche Forschungsgemeinschaft (SCHM 1151/3-1) and the National Science Foundation (NSF Award 0719445).

\section{References}

Cho PF, Poulin F, Cho-Park YA, Cho-Park IB, Chicoine JD, Lasko P, Sonenberg N (2005) A new paradigm for translational control: inhibition via $5^{\prime}-3^{\prime} \mathrm{mRNA}$ tethering by Bicoid and the eIF4E cognate 4EHP. Cell 121:411-423

Copf T, Schröder R, Averof M (2004) Ancestral role of caudal genes in axis elongation and segmentation. Proc Natl Acad Sci USA 101:17711-17716

Dubnau J, Struhl G (1996) RNA recognition and translational regulation by a homeodomain protein. Nature 379:694-699

Macdonald PM, Struhl G (1986) A molecular gradient in early Drosophila embryos and its role in specifying the body pattern. Nature 324:537-545

Mlodzik M, Gehring WJ (1987) Expression of the caudal gene in the germ line of Drosophila: formation of an RNA and protein gradient during early embryogenesis. Cell 48:465-478

Mlodzik M, Gibson G, Gehring WJ (1990) Effects of ectopic expression of caudal during Drosophila development. Development 109:271-277

Niessing D, Blanke S, Jäckle H (2002) Bicoid associates with the 5'cap-bound complex of caudal mRNA and represses translation. Genes Dev 16:2576-2582

Niessing D, Dostatni N, Jäckle H, Rivera-Pomar R (1999) Sequence interval within the PEST domain of Bicoid is important for translational repression of caudal mRNA in the anterior region of the Drosophila embryo. EMBO J 18:1966-1973

Niessing D, Driever W, Sprenger F, Taubert H, Jäckle H, Rivera-Pomar R (2000) Homeodomain position 54 specifies transcriptional versus translational control by Bicoid. Mol Cell 5:395-401

Olesnicky EC, Brent AE, Tonnes L, Walker M, Pultz MA, Leaf D, Desplan C (2006) A caudal mRNA gradient controls posterior development in the wasp Nasonia. Development 133:3973-3982

Rafiqi AM, Lemke SJ, Ferguson S, Stauber M, Schmidt-Ott U (2008) Evolutionary origin of the amnioserosa in cyclorrhapahan flies correlates with spatial and temporal expression changes of zen. Proc Natl Acad Sci USA 105:234-239

Rivera-Pomar R, Niessing D, Schmidt-Ott U, Gehring WJ, Jäckle H (1996) RNA binding and translational suppression by bicoid. Nature 379:746-749

Schulz C, Schröder R, Hausdorf B, Wolff C, Tautz D (1998) A caudal homologue in the short germ band beetle Tribolium shows similarities to both, the Drosophila and the vertebrate caudal expression patterns. Dev Genes Evol 208:283-289

Schulz C, Tautz D (1995) Zygotic caudal regulation by hunchback and its role in abdominal segment formation of the Drosophila embryo. Development 121:1023-1028

Shinmyo Y, Mito T, Matsushita T, Sarashina I, Miyawaki K, Ohuchi $\mathrm{H}$, Noji S (2005) caudal is required for gnathal and thoracic patterning and for posterior elongation in the intermediategermband cricket Gryllus bimaculatus. Mech Dev 122:231-239

Stauber M, Prell A, Schmidt-Ott U (2002) A single Hox3 gene with composite bicoid and zerknüllt expression characteristics in nonCyclorrhaphan flies. Proc Natl Acad Sci USA 99:274-279

Strimmer K, von Haesseler A (1996) Quartet puzzling: a quartet maximum-likelihood method for reconstructing tree topologies. Mol Biol Evol 13:964-969

Tautz D, Lehmann R, Schnürch H, Schuh R, Seifert E, Kienlin A, Jones K, Jäckle H (1987) Finger protein of novel structure 
encoded by hunchback, a second member of the gap class of Drosophila segmentation genes. Nature 327:383-389

Thummel C, Pirrotta V (1992) New pCaSpeR P element vectors. Dros Inf Serv 71:150

Wolff C, Schröder R, Schulz C, Tautz D, Klingler M (1998) Regulation of the Tribolium homologues of caudal and hunchback in Drosophila: evidence for maternal gradient systems in a short germ embryo. Development 125:3645-3654
Wu LH, Lengyel JA (1998) Role of caudal in hindgut specification and gastrulation suggests homology between Drosophila amnioproctodeal invagination and vertebrate blastopore. Development 125:2433-2442

Xu X, Xu PX, Suzuki Y (1994) A maternal homeobox gene, Bombyx caudal, forms both mRNA and protein concentration gradients spanning anteroposterior axis during gastrulation. Development 120:277-285 